\section{MS24-05 Electric ordering in metal-organic perovskites from total neutron scattering}

Helen D. Duncan ${ }^{1}$, Anthony E. Phillips ${ }^{1}$, Martin T. Dove ${ }^{1}$, Matthew G. Tucker ${ }^{2}$

1. Queen Mary, University of London

2. ISIS facility, Rutherford Appleton Laboratory

email: h.duncan@qmul.ac.uk

Metal-organic frameworks (MOFs) have received significant attention over the past decades due to the wide range of structures and functionalities that can be elicited through the judicious choice of starting components. Of particular interest are MOFs which display magnetic and/or electrical ordering, with applications such as ferroelectric or multiple-state memory. A common motif for establishing electric ordering in MOFs is to place organic guest ions into charged frameworks. Here we consider two such MOFs; potassium imidazolium hexacyanoferrate (PIH) (H5C3N2)2K $[\mathrm{Fe}(\mathrm{CN}) 6]$ and multiferroic dimethylammonium manganese formate (DMMnF) (CH3)2NH2[Mn(HCOO)3]. Both have perovskite-like architecture, with the organic guest ions occupying the A site. In the high-temperature phase of these materials, the guest ion is free to rotate, and the local symmetry at the site is less than the crystallographic symmetry. Upon cooling, the guest ion freezes into place accompanied by subtle distortions of the framework. Even in the high-temperature phase, guest-framework interactions are expected to lead to local ordering, concealed by the spatial averaging implicit in Bragg crystallographic analysis. To reveal this order we have used the reverse Monte Carlo algorithm to refine structural models against total neutron scattering data, modelling the onset of ordering as the phase transition is approached. As well as giving data appropriate for pair distribution function analysis, neutrons have the advantage of revealing the positions of hydrogen atoms and distinguishing clearly between carbon and nitrogen, making them sensitive to the exact configuration of these hydrogen-bonded organic cations. These models are used to understand the local interactions that give rise to phase transitions. We model the interactions between neighbouring dipoles and the role of the framework in mediating these. We also compare the distortions of the covalent FeC6 octahedra to those of the ionic KN6 in $\mathrm{PIH}$. The influences of a flexible formate framework compared to the rigid cyanide framework on guest ion dynamics are also compared. The structures of PIH and DMMnF are more complicated than materials previously reported using RMC, so these refinements and analysis demonstrate that RMC is suitable for more complex systems. It is hoped that an improved understanding of framework-guest interactions across phase transitions can be used to guide the design of future MOFs with desirable electric properties
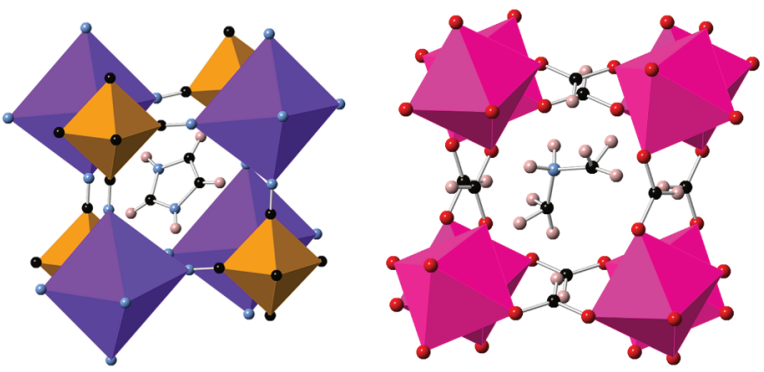

Figure 1. The perovskite-like MOFs: PIH (left) with two metal coordination environments and an imidazolium guest ion DMMnF (right) with a more flexible formate framework and dimethylammonium guest ion

Keywords: Metal-organic framework, ferroelectric, multiferroic, RMC, neutron 